\title{
Travelling waves in pipe flow
}

\author{
Holger Faisst* and Bruno Eckhardt \\ Fachbereich Physik, Philipps Universität Marburg \\ D-35032 Marburg, Germany
}

(Dated: November 7, 2018)

\begin{abstract}
A family of three-dimensional travelling waves for flow through a pipe of circular cross section is identified. The travelling waves are dominated by pairs of downstream vortices and streaks. They originate in saddle-node bifurcations at Reynolds numbers as low as 1250. All states are immediately unstable. Their dynamical significance is that they provide a skeleton for the formation of a chaotic saddle that can explain the intermittent transition to turbulence and the sensitive dependence on initial conditions in this shear flow.

PACS numbers: 47.20.Ft ,47.20.Lz,47.35.+i
\end{abstract}

Based on decades of studies it is consensus that HagenPoiseuille flow through a pipe of circular cross section belongs to the class of shear flows that does not become linearly unstable. Nevertheless, it undergoes an intermittent transition to turbulence for sufficiently high Reynolds numbers and sufficiently large initial perturbations, as first documented in the classic experiments by Reynolds [1]. Since then many studies have analyzed the mechanisms of transition and the properties of the turbulent state 2, 3, 4, 5, 6]. Particularly relevant to the present analysis are the investigations by Darbyshire and Mullin 7] which clearly show a strong sensitivity to perturbations and a broad intermittent range of decaying and turbulent perturbations in an amplitude vs. Reynolds number plane. Experimental and numerical studies [3, 8] show that the turbulent flow in the transition region is dominated by downstream vortices and streaks. Various models for their dynamics have been analyzed [9, 10]. Taking the full nonlinearity into account Waleffe developed the concept of a nonlinear turbulence cycle for the regeneration of vortices and streaks [1]. In addition, stationary solutions and travelling waves have been found in the full nonlinear equations for plane Couette, Taylor-Couette and plane Poiseuille flow 12, 13, 14, 15, 16]. It has been suggested that these structures provide a skeleton for the transition to turbulence and the observed intermittency 17, 18. They clearly dominate various observables in low Reynolds number turbulent flows [10], and are also relevant for an understanding of the effects of non-Newtonian additives [19].

The existence of exact coherent states in pipe flow has been an object of speculation for some time [5, 6, 7]. As we will show here Hagen-Poiseuille flow supports families of travelling waves with structures similar to those observed in other shear flows as well. This underlines the significance of vortex-streak interactions also in this system and opens alternative routes to modelling and controlling pipe flow.

*Electronic address: Holger.Faisst@physik.uni-marburg.de
The existence of stationary states in plane Couette flow is connected with an inversion symmetry in the laminar profile. In the absence of such a symmetry in pipe flow the simplest states we can expect are travelling waves (TWs), i.e. coherent structures that move with constant wave speed. The wave speed depends on shape and structure and is not known in advance. We search for TWs using a Newton-Raphson method applied to the full NavierStokes equations for an incompressible fluid,

$$
\begin{aligned}
\partial_{t} \mathbf{u}+(\mathbf{u} \cdot \nabla) \mathbf{u} & =\nu \Delta \mathbf{u}-\nabla p \\
\nabla \cdot \mathbf{u} & =0 .
\end{aligned}
$$

The kinematic viscosity $\nu$ is kept as a free parameter for the continuation method and the Reynolds number is determined by the mean flow velocity $U$, i.e. $R e=2 R U / \nu$, where $R$ is the pipe radius. For the numerical representation of the Navier-Stokes equation we use a FourierLegendre collocation method in cylindrical coordinates with Lagrange multipliers to account for no-slip boundary conditions at the wall and the constraints that the flow field is solenoidal, analytical and regular in the center for $r=0$. The program was verified by reproducing literature values for the linearized problem [20], for the nonlinear dynamics of optimal modes [21] and for the statistical properties of fully developed turbulent flow up to Reynolds numbers of 5000 22]. The Newton-Raphson method was implemented with a spatial resolution of $|n / 11|+|m / 11|<1$, where $n$ and $m$ are the azimuthal and downstream wavenumbers, respectively, and 56 Legendre polynomials radially. This gives us about 5600 dynamically active degrees of freedom.

Initial conditions for the Newton method are obtained by embedding pipe flow into a larger, parameter dependent family of flows. This has been successful in other cases [13, 15, 16]. For pipe flow Barnes and Kerswell [23] showed that the instabilities that arise from rotation do not extend to the case of pure pipe flow. Guided by the dominance of downstream vortices in the stationary and travelling states in planar shear flows we therefore adopted the following strategy, similar to the one used in [14]: at low Reynolds number a volume force $\mathbf{F}$ that generates a transverse flow with translation invariant downstream vortices was added. While there is no 
specific physical realization underlying this volume force, it could be achieved with differential heating and cooling as predicted by the symmetries of the states, or by magnetic forcing in an electrically conducting fluid [24]. The amplitude of the driving was then increased beyond the first bifurcation, where a stable $3-\mathrm{d}$ TW without translational symmetry appears. A path following scheme was then used to track this forced state through parameter space: the Reynolds number was increased and the amplitude of the body force decreased. If a situation with zero body force could be reached, a $3-\mathrm{d}$ TW for the original pipe flow has been found.

In this way we have identified four different states that have a discrete azimuthal rotation symmetry by construction. An $n$-fold symmetry $C_{n}$ is defined by the invariance under rotation around the pipe axis by an angle $2 \pi / n$, i.e.

$$
\left(u_{r}, u_{\phi}, u_{z}\right)(r, \phi, z) \rightarrow\left(u_{r}, u_{\phi}, u_{z}\right)(r, \phi+2 \pi / n, z) .
$$

The resolution in azimuthal direction was adjusted to the fundamental domains of angle $2 \pi / n$ to avoid carrying vanishing amplitudes.

The state that bifurcates at the lowest Reynolds number has a three-fold rotational symmetry in azimuthal direction (Fig. 11). The dynamics of the states is governed

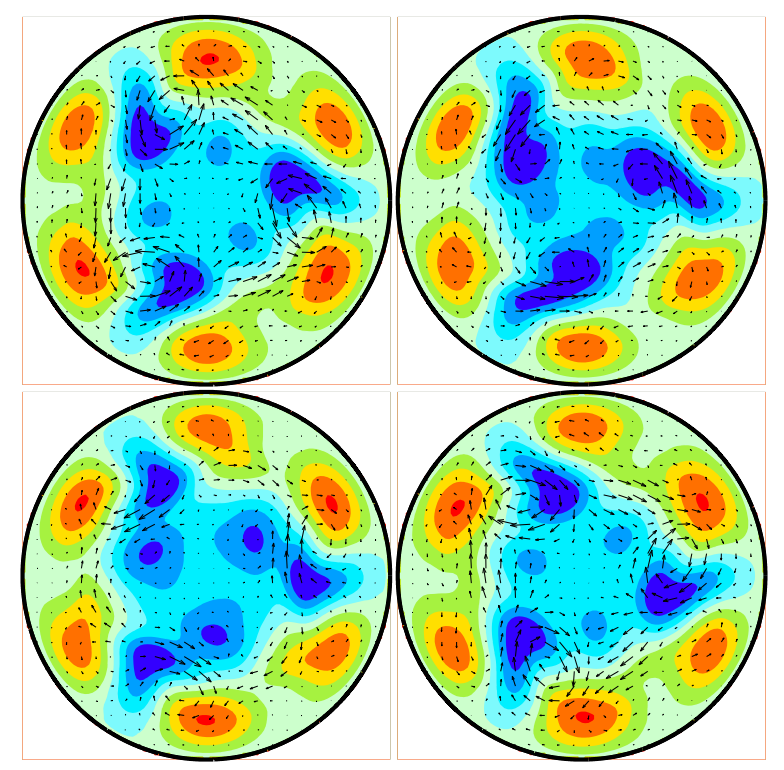

FIG. 1: Travelling wave with threefold azimuthal symmetry at the bifurcation at $R e=1250$. The frames are cross sections at different downstream positions separated by $\Delta z=L_{z} / 6$. Only half a period is shown: the last frame is the same as the first one up to a reflection at the horizontal diameter $\left(\phi \rightarrow-\phi, u_{\phi} \rightarrow-u_{\phi}\right)$. Note that the six high speed streaks near the wall move much less than the low speed streaks closer to the center. Velocity components in the plane are indicated by arrows, the downstream component by color coding: velocities faster than the parabolic profile are shown in green/yellow/red, slower ones in blue. by vortices which have a predominant downstream orientation and are slightly tilted inside the volume. They resemble near wall coherent structures in turbulent wall flows [10]. They transport slow fluid towards the center and fast fluid towards the wall, thus producing highspeed streaks near the wall and low-speed streaks towards the center (all speeds are relative to the laminar profile for that Reynolds number). The steeper gradients near the wall imply higher friction losses and higher pressure gradients for the same flow speed. The position of the fast-speed streaks hardly changes in downstream direction, whereas the low-speed streaks change shape and move in position quite a bit.

In addition to the $C_{n}$ symmetry externally imposed by the forcing the TW's dynamically develop a 'shift-andreflect' symmetry, in agreement with the streak instability of other model flows [25]: if $L_{z}$ is the wavelength of the state, then

$$
\left(u_{r}, u_{\phi}, u_{z}\right)(r, \phi, z) \rightarrow\left(u_{r},-u_{\phi}, u_{z}\right)\left(r,-\phi, z+L_{z} / 2\right) .
$$

The same shift-and-reflect symmetry is present in 3-d coherent states in plane Couette, Taylor-Couette and plane Poiseuille flow 13, 14, 15, 16].

Each TW is part of a two-parameter family of states that exists for a range of Reynolds numbers and a range of downstream wavelengths (Fig. 2). The lowest critical

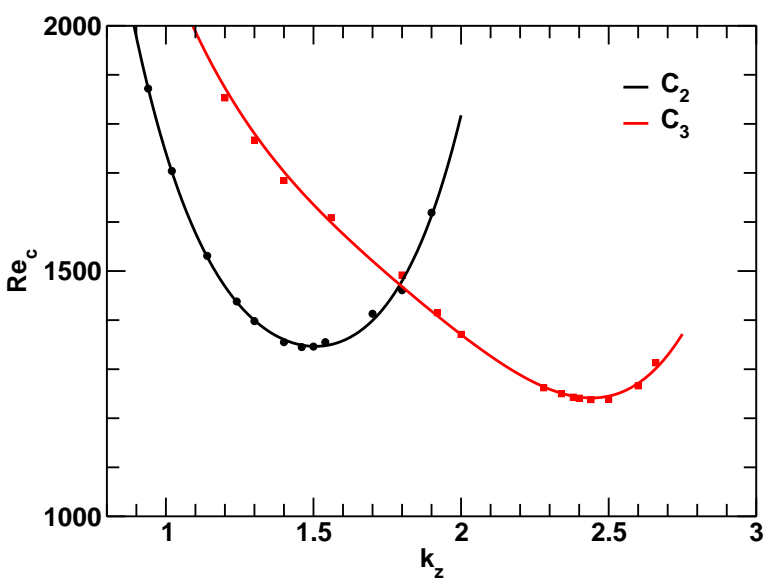

FIG. 2: Dependence of the critical Reynolds number on the downstream wavenumber for the two-, three-, and fourfold symmetric state. The numerical values (dots) are interpolated by a 4 th-order polynomial fit (continuous lines).

Reynolds number is obtained for a wavelength of about $4.2 R$ for the twofold symmetry and about $2.5 R$ for the three- and fourfold symmetric state. This period seems a bit shorter than that for optimal states in plane Couette flow where the optimal wavelength is about $2 \pi d$ with $d$ the gap width. However, when the wedge shape of the boundary is taken into account and the lengths are compared to the widths, the comparison is more favorable: the ratio widths to lengths is about $2 \pi: 4 \pi=1: 2$ in plane Couette flow and about 1:2.5 in pipe flow. 
Other states that we could identify in pipe flow include those with twofold, fourfold and fivefold symmetry (Fig. 3). All these states have a similar topology: they

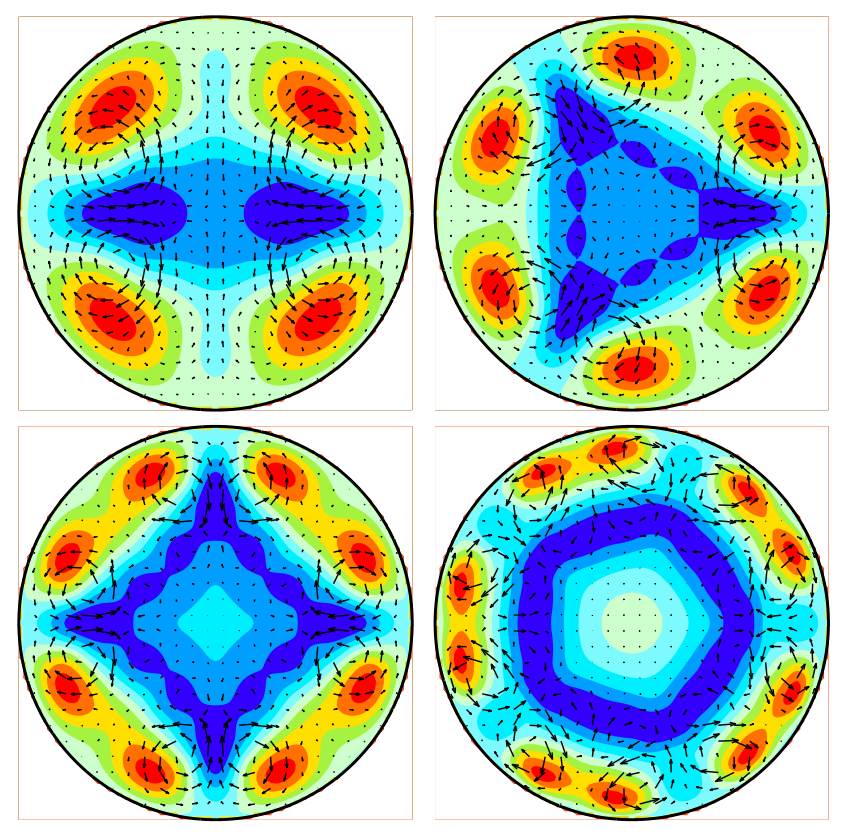

FIG. 3: Travelling waves with symmetries $C_{n}$ with $n=2,3$, 4 , and 5. In order to highlight the topology of the states all states are averaged in downstream direction. The representation of the velocity field by vectors (in-plane motion) and color (downstream component) is as in Fig. 1] The absolute scale for the velocity fields is given in Table

have $2 n$ streaks of fast fluid close to the wall and streaks of slow fluid towards the center. The high-speed streaks near the wall remain fairly stationary over one period of the wave, and the low speed streaks in the center oscillate vigorously. All states have the shift-and-reflect symmetry (1). The wavelength that gives the lowest critical Reynolds number for these states varies with the symmetry (Fig. 2). Several properties of these states are listed in Table \. The lowest critical Reynolds number is obtained for the TW with $C_{3}$ symmetry, for which the arrangement of vortices is optimal in the sense of being closest to a hexagonal packing, the preferred pattern in other systems. The states with $C_{4}$ and $C_{5}$ symmetry are not included in the table as they seem to be the ones which are most sensitive to the numerical resolution and not fully converged. A preliminary estimate for their critical Reynolds number is about 1590 for $C_{4}$ and 2600 for $C_{5}$.

Interestingly, the list of TWs does not include a state with the $C_{1}$ - symmetry of the mode that shows the strongest linear transient growth [20, 26]. A state with $C_{1}$ symmetry could be found for non-vanishing volume force, but up to Reynolds numbers of 4000 it could not be continued to pure pipe flow without reconnecting to the laminar profile. This should have implications for low dimensional model building [6, 27] where linear arguments have led to an emphasis of the $C_{1}$ mode.

\begin{tabular}{ccc} 
symmetry & \multicolumn{1}{c}{$C_{2}$} & \multicolumn{1}{c}{$C_{3}$} \\
\hline \hline$R e_{c}$ & 1350 & 1250 \\
$L_{c} / R$ & 4.19 & 2.58 \\
$c / U$ & 1.43 & 1.29 \\
$n_{u}$ & 2 & 1 \\
$u_{s} / U$ & 0.38 & 0.35 \\
$u_{t} / U$ & 0.035 & 0.046
\end{tabular}

TABLE I: Selected properties of travelling waves at the saddle-node bifurcation. Given is the critical Reynolds number $R e_{c}$ at the optimal wavelength $L_{c}$, the phase velocity $c$ and the number $n_{u}$ of unstable dimensions. $u_{s}$ is the maximum deviation of the streamwise velocity from the laminar flow, $u_{t}$ is the maximum transverse velocity component.

The phase speed $c$ of the TWs is a function of both parameters, length and Reynolds number. In all cases the phase speed is larger than the mean speed but slower than the maximal speed possible with a laminar fluid (Fig. (4). That is to say, the wave still propagates downstream when viewed from a frame of reference moving with the mean flow velocity.

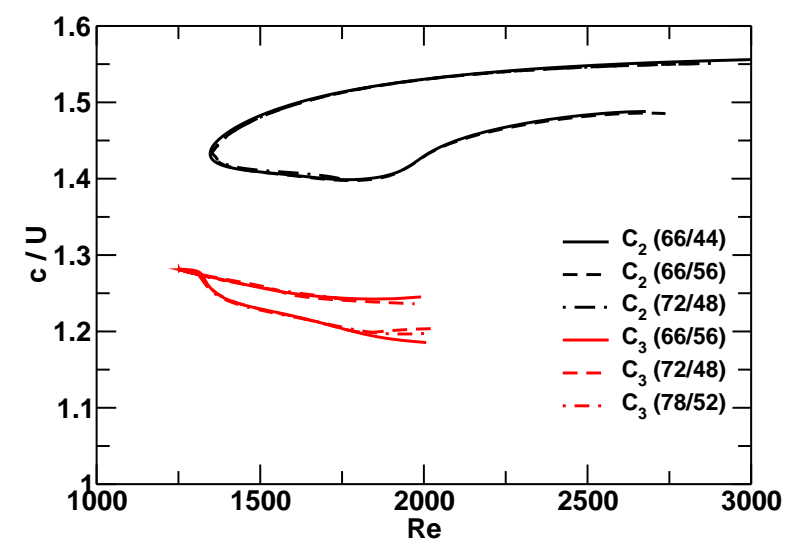

FIG. 4: Phase velocities for the travelling states at optimal wavelength as a function of Reynolds number. The velocities are normalized by the mean downstream velocity for the states. Three different resolutions are plotted, indicated by $(J / K)$ where $J$ is the number of independent Fourier modes, $K$ is the number of Legendre polynomials.

By advecting the unperturbed profile small transverse components can produce strong downstream streaks. Table \shows that for the TWs the transverse components are about an order of magnitude smaller than the differences between the downstream components of the laminar profile and the TW.

The TWs appear as finite amplitude saddle-node bifurcations. Away from the bifurcations there is an upper (nodal) and a lower (saddle) branch. The saddle has one more positive eigenvalue than the node, but in all cases we found that even at the point of bifurcation at least one eigenvalue is positive: all states are thus unstable right from the point of creation. This is in contrast to plane Couette flow, where at least the lowest state has 
an interval of stability, although a tiny one [13].

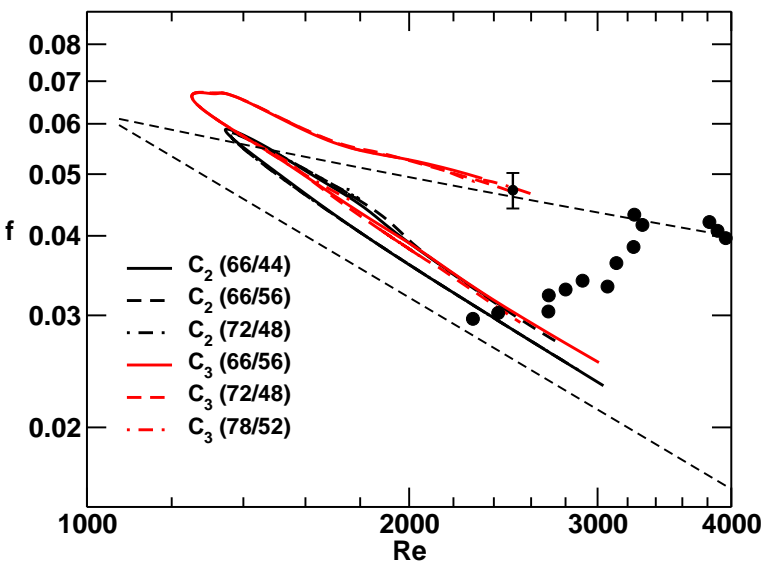

FIG. 5: Travelling waves and turbulent states in pipe flow. The friction factor as defined in Eq. (2) is shown vs. Reynolds number. The TWs are labeled by their azimuthal symmetries. Three different resolutions are plotted as explained in Fig. 4 The lower dashed line indicates the strict lower bound from the laminar profile (where $f=64 / R e$ ), the upper dashed line gives the mean friction factor from a logarithmic profile. The full circles are experimental data from [28] or from our numerical simulations (the value at $R e=2500$ with error bars).

The friction properties of all states together with experimental and numerical results for turbulent flows are shown in Fig. [5] The friction factor $f$ is defined as

$$
f=4 R \Delta p / \rho U^{2} L_{z}
$$

with $\Delta p$ the pressure drop over a distance $L_{z}$ along the pipe and $\rho$ the density of the fluid. The saddle-node bifurcations take place at Reynolds numbers significantly below the values where typical perturbations induce turbulent dynamics. At the bifurcation the friction factor is higher than the value extrapolated from turbulent states at higher Re. The upper branches in Fig. [5]correspond to the lower branches in Fig. 4 states with higher friction have lower phase velocity and vice versa.

Besides the TWs presented here we can expect many other TWs. On the assumption that narrower vortices will require stronger driving we anticipate that they will have critical Reynolds number higher than the 1250 found for the $C_{3}$ state. In addition, there are all the states that bifurcate from the TWs, as well as states that bifurcate in secondary bifurcations. Furthermore, there will be homoclinic and heteroclinic connections between these TWs as well as other periodic and aperiodic states. All these states together then form a chaotic saddle in phase space that supports the turbulent dynamics 29]. One characteristic of this chaotic saddle is an exponential distribution of life times and this is indeed found in numerical simulations [30]. It will be interesting to see to which extent these states can be used to approximate properties of the turbulent flow.

Support by the Deutsche Forschungsgemeinschaft is greatfully acknowledged.
[1] O. Reynolds. Phil. Trans. R. Soc. 174, 935 (1883).

[2] I.J. Wygnanski, F.H. Champagne. J. Fluid Mech. 59, 281 (1973). I.J. Wygnanski, M. Sokolov, D. Friedman. J. Fluid Mech. 69, 283 (1975).

[3] S. Eliahou, A. Tumin, I. Wygnanski. J. Fluid Mech. 361, 333 (1998). G. Han, A. Tumin, I. Wygnanski. J. Fluid Mech. 419, 1 (2000). H. Shan, B. Ma, Z. Zhang, F.T.M. Nieuwstadt. J. Fluid Mech. 387, 39 (1999).

[4] B. Ma, C.W. van Doorne, Z. Zhang, F.T.M. Nieuwstadt. J. Fluid Mech. 398, 181 (1999).

[5] L. Boberg, U. Brosa. Z. Naturforsch. 43a, 697 (1988).

[6] U. Brosa, S. Grossmann. Eur. Phys. J. B 9, 343 (1999).

[7] A.G. Darbyshire, T. Mullin. J. Fluid Mech. 289, 83 (1995).

[8] P.J. Schmid, D.S. Henningson. Stability and Transition in Shear Flows, Springer, (2001)

[9] L.N. Trefethen, A.E. Trefethen, S.C. Reddy, T.A. Driscol. Science 261, 578 (1993). S. Grossmann. Rev. Mod. Phys. 72, 603 (2000).

[10] P. Holmes, J.L. Lumley, G. Berkooz. Turbulence, Coherent Structures, Dynamical Systems and Symmetry. Cambridge University Press, (1996).

[11] J.M. Hamilton, J. Kim, F. Waleffe. J. Fluid Mech. 287,
317 (1995). F. Waleffe. Phys. Fluids 7, 3060 (1995). F. Waleffe. Phys. Fluids 9, 883 (1997).

[12] A. Cherhabili, U. Ehrenstein. J. Fluid Mech. 342, 159 (1997).

[13] M. Nagata; J. Fluid Mech. 217, 519 (1990). R.M. Clever, F.H. Busse. J. Fluid Mech. 344, 137 (1997). M. Nagata. Phys. Rev. E 55(2), 2023 (1997).

[14] F. Waleffe. Phys. Rev. Lett. 81, 4140 (1998).

[15] H. Faisst, B. Eckhardt. Phys. Rev. E 61, 7227 (2000).

[16] F. Waleffe. J. Fluid Mech. 435, 93 (2001).

[17] A. Schmiegel, B. Eckhardt. Phys. Rev. Lett. 79, 5250 (1997).

[18] B. Eckhardt, H. Faisst, J. Schumacher and A. Schmiegel. Advances in turbulence IX, I.P. Castro, P.E. Hanock and T.G. Thomas (eds), Barcelona, 701 (2002)

[19] P.A. Stone, F. Waleffe, M.D. Graham. Phys. Rev. Lett. 89, 208301 (2002).

[20] P.J. Schmid, D.S. Henningson. J. Fluid Mech. 277, 197 (1994).

[21] O.Y. Zikanov. Phys. Fluids 8, 2923 (1996).

[22] J.G.M. Eggels, F. Unger, M.H. Weiss, J. Westerweel, R.J. Adrian, R. Friedrich, F.T.M. Nieuwstadt. J. Fluid Mech. 268, 175 (1994). M. Quadrio, S. Sibilla. J. Fluid 
Mech. 424, 217 (2000).

[23] D.R. Barnes, R.R. Kerswell. J. Fluid Mech. 417, 103 (2000).

[24] U. Brosa. Z. Naturforsch. 46a, 473 (1991).

[25] F. Waleffe. Stud. Appl. Math. 95, 319 (1995).

[26] L. Bergström. Phys. Fluids A 5, 2710 (1993).

[27] L. Bergström. Eur. J. Mech. B 18, 635 (1999).

[28] H. Schlichting. Boundary-Layer Theory. New York:
McGraw-Hill, (1979).

[29] T. Tél. Directions in Chaos, Vol. 3, ed. Hao, B.-L. (World Scientific, Singapore) (1990), pp. 149-221.

[30] H. Faisst, B. Eckhardt. Sensitive dependence on initial conditions in transition to turbulence in pipe flow, to be published 